\title{
Annealing-induced reduction in nanoscale heterogeneity of thermally evaporated amorphous $\mathrm{As}_{2} \mathrm{~S}_{3}$ films
}

\author{
A. C. Y. Liu, ${ }^{1, a)}$ Xidong Chen, ${ }^{2}$ D.-Y. Choi, ${ }^{3}$ and B. Luther-Davies ${ }^{3}$ \\ ${ }_{1}^{1}$ Department of Materials Engineering, Monash University, Clayton, Victoria, 3800, Australia \\ ${ }^{2}$ Materials Science Division, Argonne National Laboratory, Illinois 60439-4845, USA and Cedarville \\ University, Cedarville, Ohio 45314, USA \\ ${ }^{3}$ Centre for Ultrahigh Bandwidth Devices for Optical Systems, Laser Physics Centre, Research School of \\ Physical Science and Engineering, Australian National University, ACT, 0200, Australia
}

(Received 27 May 2008; accepted 17 September 2008; published online 10 November 2008)

\begin{abstract}
The morphology and structural order of thermally deposited and annealed amorphous $\mathrm{As}_{2} \mathrm{~S}_{3}$ films have been investigated using high resolution transmission electron microscopy. It was found that both the as-deposited and annealed films contained sparsely distributed nanocrystallites of the orpiment $\mathrm{As}_{2} \mathrm{~S}_{3}$ crystalline phase. However, from selected area electron diffraction both films appeared amorphous. Fluctuation electron microscopy revealed that the as-deposited film contained greater nanoscale inhomogeneity. Low temperature annealing reduced the nanoscale inhomogeneity and resulted in a more homogeneous and energetically favorable network. The reduction in nanoscale inhomogeneity upon low temperature annealing was accompanied by the appearance of a first sharp diffraction peak in the diffraction pattern. This first-sharp diffraction peak has been attributed to chemical ordering of interstitial voids. Our measurements suggest that this chemical short-range ordering is associated with the dissolution of the energetically unfavorable larger correlated structures that contribute to the inhomogeneity of the as-deposited film.
\end{abstract}

(C) 2008 American Institute of Physics. [DOI: 10.1063/1.3009971]

\section{INTRODUCTION}

Chalcogenide glasses possess high refractive indices and large third order nonlinear optical coefficients making them compelling candidates for waveguides and all-optical devices. ${ }^{1}$ The inherent structural flexibility of the chalcogenide network results in a plethora of photoinduced structural changes such as photocrystallization, photoamorphization, photodecomposition, photocontraction or expansion, photovaporization, photodissolution of metals, and photoinduced changes in local atomic configurations. ${ }^{1,2}$ These changes in local atomic configurations are accompanied by changes in the optical constants of the material; in particular shifts in the optical band gap result in photobleaching or photodarkening. ${ }^{2}$ The susceptibility of the chalcogenide glasses to light-induced structural change can be used to fabricate diffraction gratings, waveguides, and fibers and suggests the use of chalcogenide glasses as inorganic photoresists. ${ }^{1,3}$

Recently, significant progress has been made in reducing optical losses due to scattering off rough waveguide walls in etched amorphous $\mathrm{As}_{2} \mathrm{~S}_{3}$ waveguides, permitting waveguide fabrication with losses as low as $0.05 \mathrm{~dB} / \mathrm{cm}^{4}$ The waveguides were produced from thermally evaporated films subjected to low temperature annealing and then photolithography and plasma reactive ion etching. ${ }^{4}$ Roughening of the side walls is an intrinsic problem in the processing of such devices as the inherent nanoscale phase separation of the glass $^{5}$ is thought to result in variable etch rates, giving a

${ }^{\text {a)} E l e c t r o n i c ~ m a i l: ~ a m e l i a . l i u @ e n g . m o n a s h . e d u . a u . ~}$ rough final surface. ${ }^{6}$ This surface roughness and resultant optical losses may place an ultimate limit on the loss of waveguides made from $\mathrm{As}_{2} \mathrm{~S}_{3}$ films.

Here we investigate the morphology and structure of thermally evaporated $\mathrm{As}_{2} \mathrm{~S}_{3}$ films in their as-deposited and thermally annealed states using transmission electron microscopy (TEM). Our studies suggest that the as-deposited films have significantly more nanoscale heterogeneity than the annealed films. This finding supports previous studies on process optimization, where the inclusion of an annealing step created a more homogeneous glass and reduced optical losses due to surface scattering off etched surfaces.

\section{EXPERIMENT}

$\mathrm{As}_{2} \mathrm{~S}_{3}$ films $(40 \mathrm{~nm})$ were deposited using a commercial glass (Amorphous Materials, Garland, Texas, USA) and a tungsten boat in a thermal evaporator pumped to a base pressure of $3 \times 10^{-7}$ Torr. The boat temperature was maintained at $315{ }^{\circ} \mathrm{C}$ to give a constant deposition rate for a given source/substrate distance. ${ }^{4}$ The substrate used was high purity single crystal $\mathrm{NaCl}$ (Structure Probe Inc., Westchester, Pennsylvania, USA), freshly cleaved just prior to introduction into the deposition chamber. Part of the film was thermally annealed on the substrate under vacuum at a temperature of $130{ }^{\circ} \mathrm{C}$ for $24 \mathrm{~h}$. The films were floated off the substrate in de-ionized water and picked up by copper TEM grids for study in the microscope.

TEM was performed on a Jeol JEM4000EX microscope operated at $200 \mathrm{keV}$. Bright-field and high resolution TEM (HRTEM) images and diffraction patterns were recorded using a Gatan slow scan charge coupled device camera. Fluc- 
tuation electron microscopy (FEM) was also performed on this same microscope using the tilted dark-field technique. ${ }^{7,8}$ Briefly, an automated script was used to acquire a series of tilted dark-field images with a range of scattering vectors of $1.2-10 \mathrm{~nm}^{-1}$. The images were filtered to remove noise, distortions, and long-range intensity variations, and the intensity variance was calculated to yield the intensity variance as a function of scattering vector $k$. Here $k$ is projected onto the diffraction plane and expressed in $\mathrm{nm}^{-1}$. The intensity variance is related to the pair-pair atomic correlations in the material and accesses order in the range of medium-range order (MRO) (1-3 nm). ${ }^{9}$ The dark-field technique is a way of statistically sampling the scattering from different regions in the material. If there is a large difference between the scattering in adjacent regions (high intensity variance) then the material contains regions of correlated structure. ${ }^{10}$ We note that the technique as employed is not fully quantitative and can only provide relative measures of MRO between materials of similar composition and density and from specimen areas of the same thickness. ${ }^{7}$

Specimens were found to charge slightly in the electron beam. Stable areas were discovered near the copper grid bars. Two different specimens of both the as-deposited and annealed films were examined; the FEM results are the average of ten regions taken from the two respective specimens studied.

Traditional techniques to prepare bulk samples or thick films for study in the TEM include a first mechanical polishing step and then ion milling with $\mathrm{keV}$ ions or chemical etching to obtain electron transparency in a few small specimen areas. In this case deposition of thin films onto rock salt and then floating the films off to provide free-standing specimens to examine in the microscope was preferred for several reasons. First, transient heating and the collision cascades associated with ion milling could introduce unacceptable levels of structural modification into these sensitive materials. Second, the mechanical polishing and ion sputtering of an As-based material poses significant occupational health, safety, and environmental contamination risks. Lastly, the technique of FEM is very demanding experimentally and to obtain good statistics in the measurements many large areas $\left(200 \times 200 \mathrm{~nm}^{2}\right)$ that have the same thickness and are of uniform thickness must be found. Chemical etching would result in too great a degree of surface roughness to permit study with FEM.

Given such considerations we did not perform the same TEM analyses on the bulk glass. To link our results with the structure of the bulk glass, we performed Raman spectroscopy on thicker films in the as-deposited and annealed states and also the bulk glass. We note that Raman spectroscopy was not possible on the thin films studied in the TEM as the signal from the actual film was too small to give an acceptable signal-to-noise ratio. Raman spectra were recorded using a micro-Raman system in backscattering geometry with an $808 \mathrm{~nm}$ source laser. The spectral resolution was $3 \mathrm{~cm}^{-1}$. We measured the spectra of both fresh samples and samples after $20 \mathrm{~min}$ of laser irradiation employing different laser powers. When no difference between these two spectra was apparent, we considered any photoinduced structural change
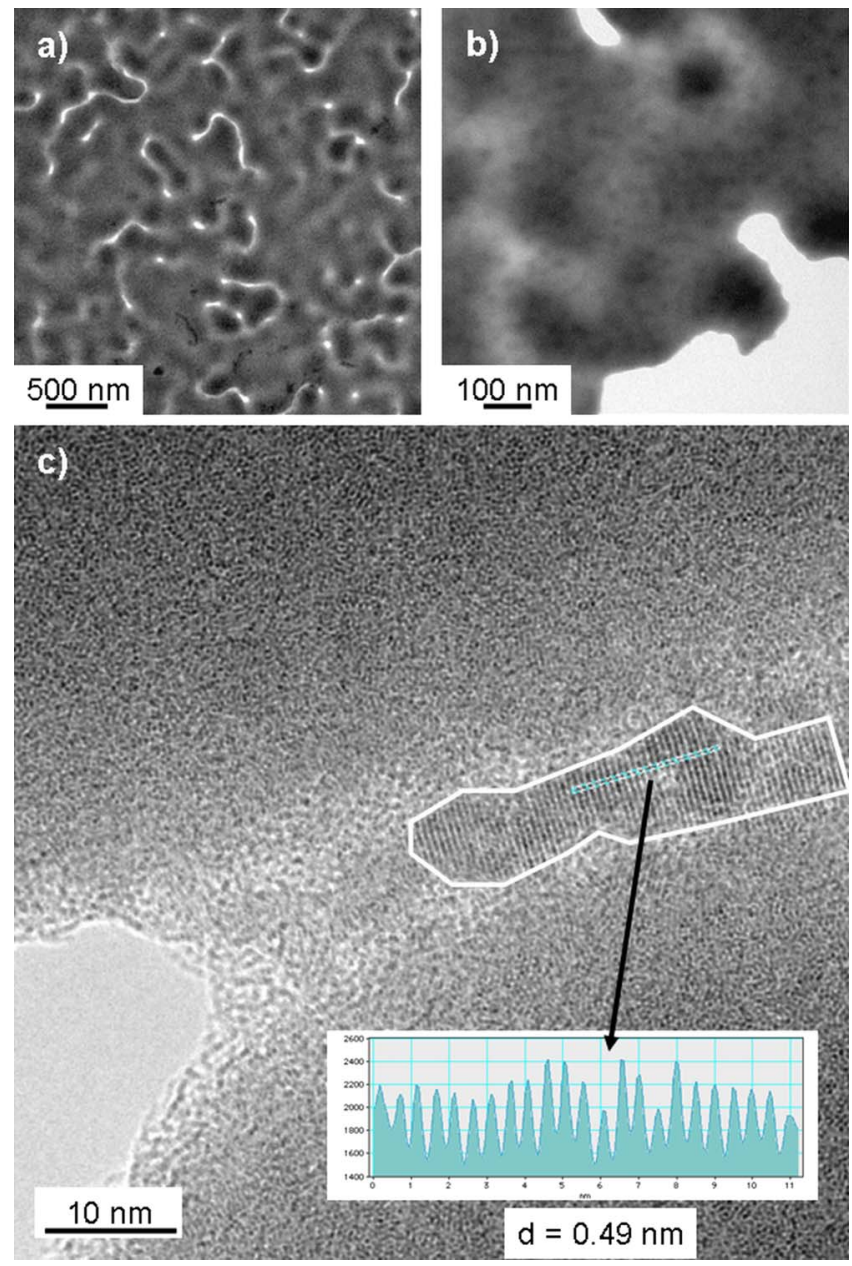

FIG. 1. (Color online) (a) Low and (b) intermediate magnification brightfield images of an as-deposited $\mathrm{As}_{2} \mathrm{~S}_{3}$ film. (c) HRTEM image of an asdeposited $\mathrm{As}_{2} \mathrm{~S}_{3}$ film showing a $30 \times 10 \mathrm{~nm}^{2}$ crystallite with lattice fringes corresponding to a $d$-spacing of $0.49 \mathrm{~nm}$.

to be suppressed. In this case, the laser power was below $50 \mathrm{~mW} / \mathrm{cm}^{2}$, and the typical collection time was $5 \mathrm{~s}$ for all the spectra.

\section{RESULTS}

Figure 1 shows a series of images at increasing magnification of the as-deposited film. In the low magnification image [Fig. 1(a)] we clearly see a large scale domain morphology that has been found typical for such evaporated thin chalcogenide films. ${ }^{11}$ The contrast is due to thickness variations in the material corresponding to hills and valleys. In the intermediate magnification image [Fig. 1(b)], we see that the material itself has a mottled contrast with lighter and darker regions. In high resolution [Fig. 1(c)] and in thin areas of the film, regions containing lattice fringes could be found. The periodicity of the fringes in this image determined from a line profile was found to be $0.49 \mathrm{~nm}$ and the projected extent of the crystallite was estimated at $30 \times 10 \mathrm{~nm}$.

Figure 2 shows a similar series of images for the annealed film. It is apparent from the low magnification image [Fig. 2(a)] that the morphology is considerably altered, perhaps due to tensile stresses during annealing from differences in the thermal expansion coefficient of film and substrate. 

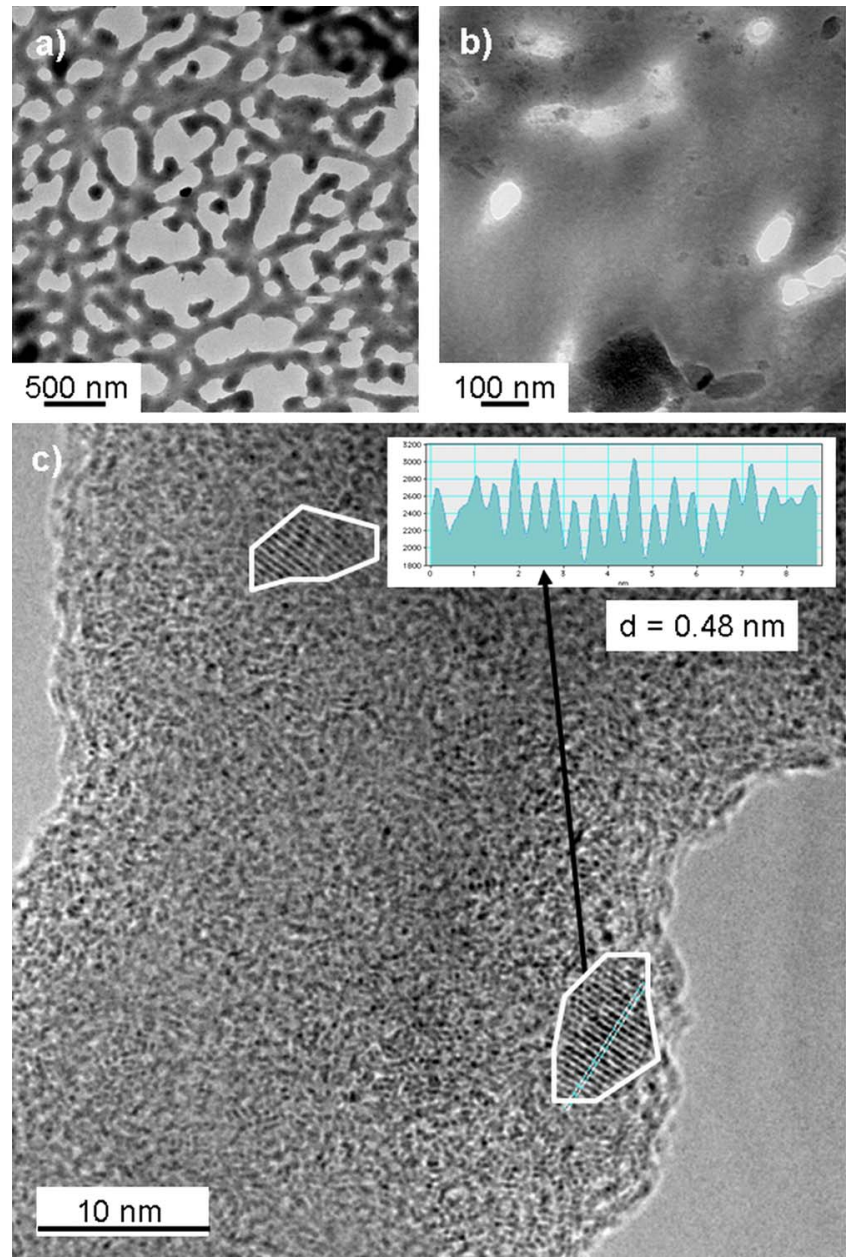

FIG. 2. (Color online) (a) Low and (b) intermediate magnification brightfield images of an annealed $\mathrm{As}_{2} \mathrm{~S}_{3}$ film. (c) HRTEM image of an annealed $\mathrm{As}_{2} \mathrm{~S}_{3}$ film showing $10 \times 5 \mathrm{~nm}^{2}$ crystallites with lattice fringes corresponding to a $d$-spacing of $0.48 \mathrm{~nm}$.

The intermediate magnification image [Fig. 2(b)] shows a distinct decrease in the mottled appearance of the material (except for the large dark pieces of dust which were avoided). In the high resolution image in a thin area of the film [Fig. 2(c)], small crystallites of $10 \times 5 \mathrm{~nm}^{2}$ in projected dimensions can be detected with a lattice periodicity in this case of $0.48 \mathrm{~nm}$.

The specimens were surveyed and a range of the sparsely distributed crystallites were imaged using HRTEM for both the as-deposited and annealed $\mathrm{As}_{2} \mathrm{~S}_{3}$ films. The periodicities of the lattice fringes are summarized in a histogram in Fig. 3. The uncertainty in the fringe spacings is indicated by a bar underneath the legend. Interplanar spacings for the realgar (AsS) and orpiment $\left(\mathrm{As}_{2} \mathrm{~S}_{3}\right)$ phases are shown by lines on the graph. ${ }^{12,13}$ It is clear that most of the measured lattice spacings correspond well to the low index planes of orpiment. In contrast, the realgar phase has only one $d$-spacing within error of the observed interplanar spacings.

Figures 4(a) and 4(b) show the selected area diffraction patterns taken from the as-deposited and annealed $\mathrm{As}_{2} \mathrm{~S}_{3}$ films, respectively. Here the scattering vector $q=2 \sin (\theta) / \lambda$. There are clearly large differences between the two patterns.

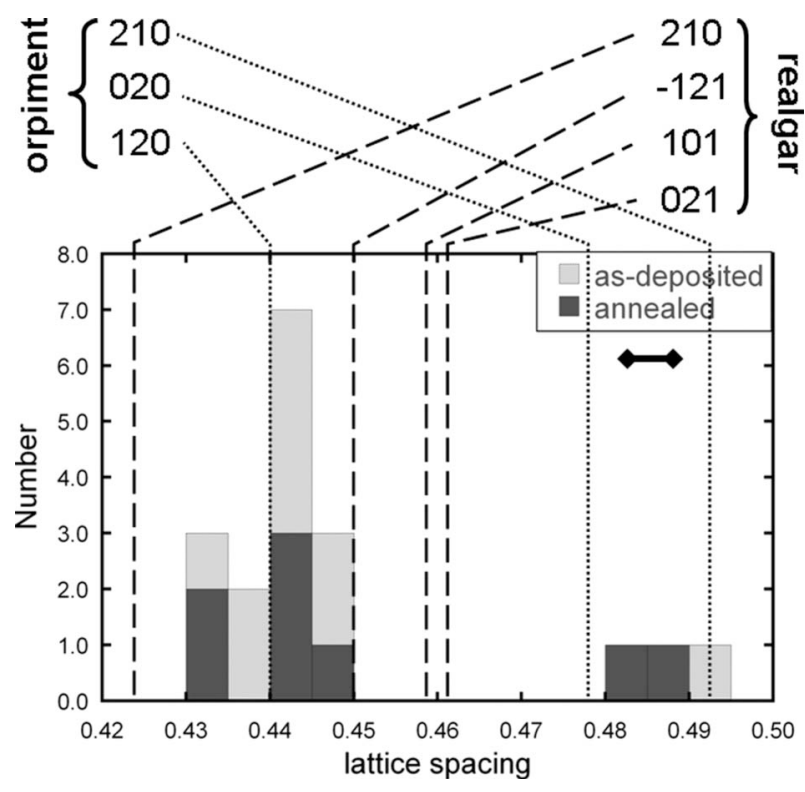

FIG. 3. Histogram of the periodicity of the lattice fringes found in the as-deposited and annealed $\mathrm{As}_{2} \mathrm{~S}_{3}$ films. $d$-spacings for orpiment (Ref. 12) and realgar (Ref. 13) in the range $0.42-0.50 \mathrm{~nm}$.

Inset to each pattern is an enlarged section of the first broad diffraction maximum. The as-deposited diffraction pattern has a slightly grainier intensity distribution than the diffraction pattern from the annealed sample, consistent with the
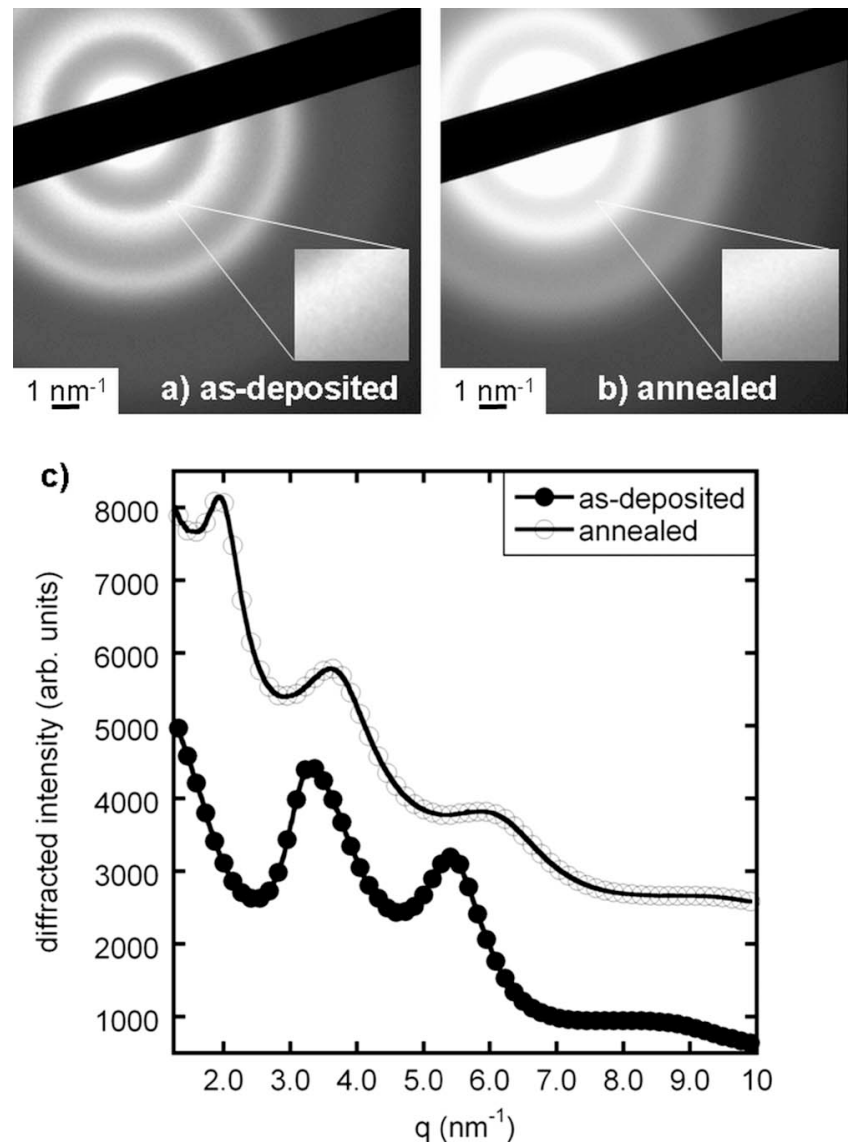

FIG. 4. Selected area diffraction patterns of (a) the as-deposited and (b) thermally annealed $\mathrm{As}_{2} \mathrm{~S}_{3}$ films. (c) Azimuthal averages of the diffraction patterns displayed in (a) and (b), $q=2 \sin (\theta) / \lambda$. 

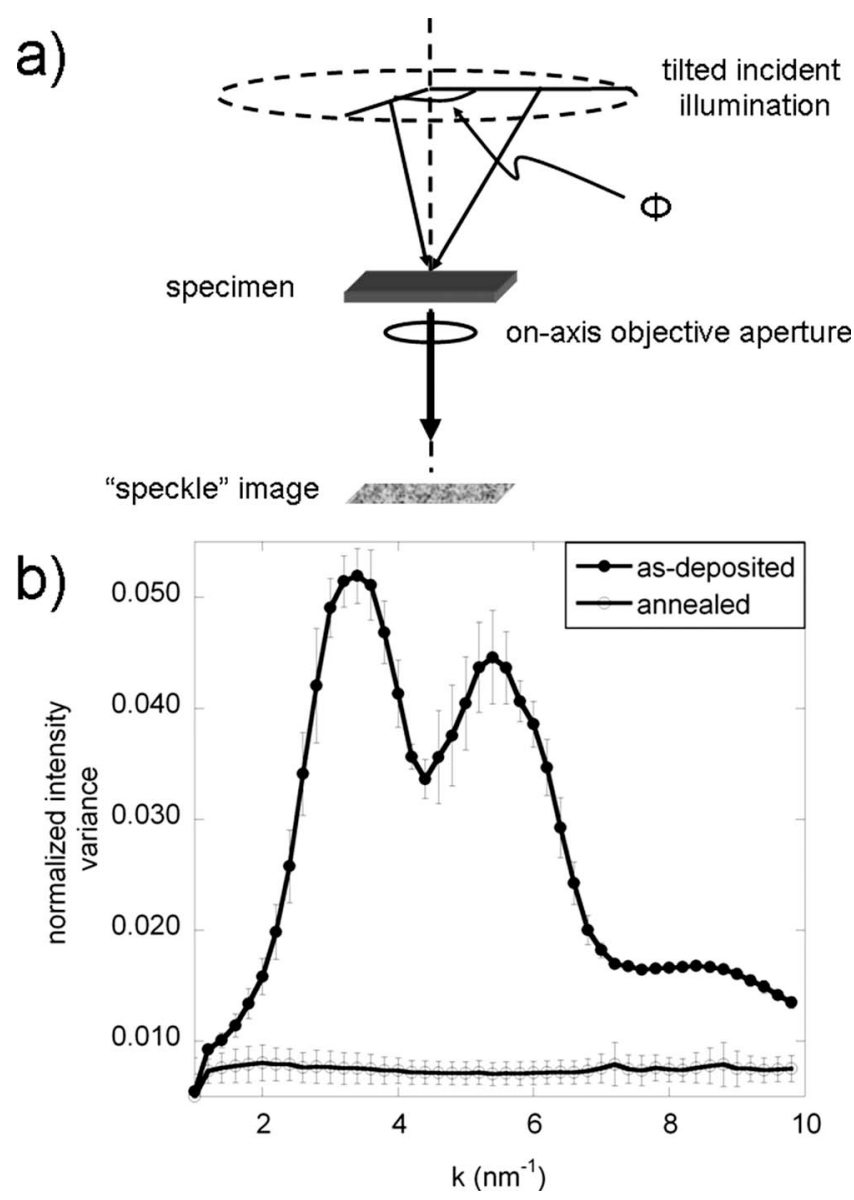

FIG. 5. (a) Experimental geometry for tilted dark-field FEM. Scan direction can be changed to probe for anisotropies in structure. (b) Averaged $V(k)$ plots from the as-deposited and annealed $\mathrm{As}_{2} \mathrm{~S}_{3}$ films.

mottled contrast of the bright-field image. Despite finding nanocrystallites in both specimens using HRTEM, the diffraction patterns from an areal average of $\sim 200 \mathrm{~nm}$ in diameter results in a diffraction pattern with diffuse rings characteristic of an amorphous material. Figure 4(c) displays the azimuthal average of selected area diffraction patterns taken from a slice in the angular range of $60^{\circ}-80^{\circ}$. Annealing is seen to result in a shift in the peak positions to higher wave vector and also in the appearance of a first-sharp diffraction peak (FSDP) at $2 \mathrm{~nm}^{-1}$. The peak positions compare well to previous $\mathrm{x}$-ray diffraction studies of thermally evaporated $\mathrm{As}_{2} \mathrm{~S}_{3}$ films. However, in the previous studies, the FSDP was present in the diffraction pattern of the as-deposited film and decreased in magnitude with annealing, opposite to our findings. ${ }^{14,15}$

Ten different areas from two different specimens were examined with FEM for both the as-deposited and annealed $\mathrm{As}_{2} \mathrm{~S}_{3}$ films. In the tilted dark-field implementation of FEM, the incident parallel electron beam is incrementally tilted in one direction to obtain a series of dark-field images. A small objective aperture in the back-focal plane selects the on-axis scattered electrons to make the dark-field image as shown in Fig. 5(a). From this procedure one obtains a plot of variance as a function of scattering vector $V(k)$. Most of the tilted dark-field series were obtained with a nominal scanning direction of $\phi=0^{\circ}$ i.e., along one azimuthal direction. In some

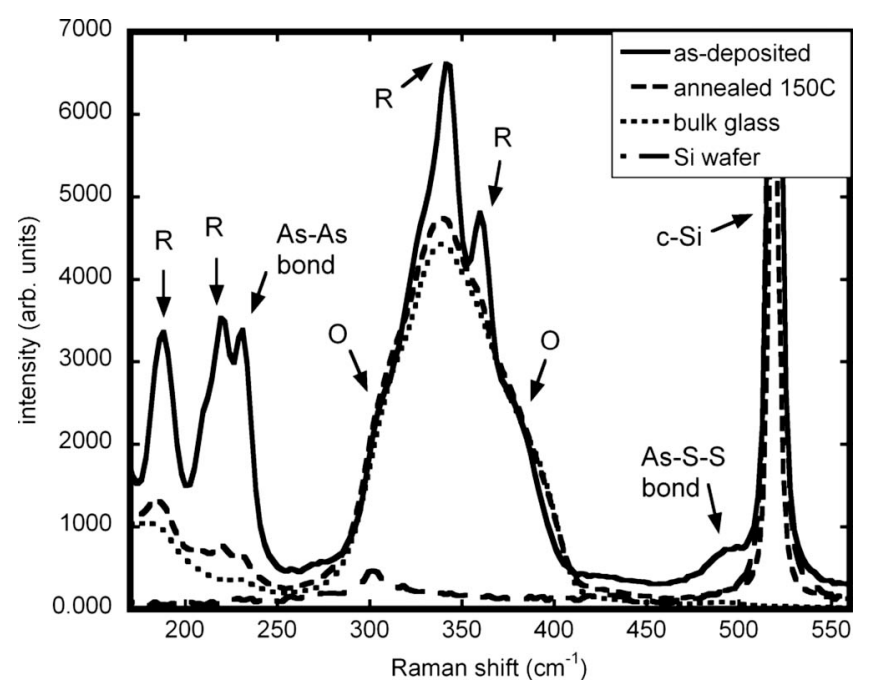

FIG. 6. Raman spectra of the bulk glass (solid line) and thick $\mathrm{As}_{2} \mathrm{~S}_{3}$ films in the as-deposited (dashed line) and thermally annealed (dotted line) states. A reference spectrum from the crystalline Si substrate is shown (long dashes). The peak assignments are discussed in the text.

areas the tilting direction was changed to $\phi=45^{\circ}$ or $\phi=90^{\circ}$ with respect to the initial nominal $\phi=0^{\circ}$ direction. Varying the tilting direction in this way will provide sensitivity to possible orientation biases in the regions of the correlated structure detected with FEM. In this case, both for the asdeposited and annealed $\mathrm{As}_{2} \mathrm{~S}_{3}$ films, there was no significant difference between the $V(k)$ plots obtained from different areas and with different scanning directions. Given this, the FEM data sets from the as-deposited and annealed $A s_{2} S_{3}$ films were averaged and presented in Fig. 5(b). The error is the standard error in the measurements. It is clear that the as-deposited film displays a much higher and much more featured intensity variance in the range of the $k$ studied than the film that was subject to the low temperature anneal.

Figure 6 displays Raman spectra from bulk glass and from thick thermally evaporated $\mathrm{As}_{2} \mathrm{~S}_{3}$ films $(\sim 15 \mu \mathrm{m})$ in the as-deposited and annealed $\left(150{ }^{\circ} \mathrm{C}\right.$ for $\left.24 \mathrm{~h}\right)$ states. The spectra have been vertically scaled so that the magnitudes of the peaks at $350 \mathrm{~cm}^{-1}$ coincide. A reference spectrum from the crystalline $\mathrm{Si}$ substrate is also displayed, demonstrating that the sharp peak at $520 \mathrm{~cm}^{-1}$ is due to the substrate. From examining the spectra it is clear that the spectrum from the as-deposited film is much more highly featured than the spectra from either the annealed film or the bulk glass. Peaks in this spectrum correspond to modes from $\mathrm{As}_{4} \mathrm{~S}_{4}$ monomers (labeled " $\mathrm{R}$ ") that have been broadened when compared to the same modes in crystalline realgar. ${ }^{5}$ There are also peaks due to $\mathrm{As}\left(\mathrm{S}_{1 / 2}\right)_{3}$ pyramidal units (labeled "O"), broadened and blueshifted when compared to the same modes in crystalline orpiment. ${ }^{5}$ Other peaks are the peak at $234 \mathrm{~cm}^{-1}$ that has been attributed to As-As bonds in the glass network and the weak peak at $495 \mathrm{~cm}^{-1}$ due to the As-S-S bond. ${ }^{16}$ It is clear from the Raman spectra that annealing the film resulted in a structure that more greatly resembles that of the bulk glass.

\section{DISCUSSION}

The domain morphology of thermally evaporated chalcogenide glasses has been studied in depth for examples such 
as $\mathrm{As}_{2+x} \mathrm{Se}_{3-x}(x=0, \pm 0.025, \pm 0.05, \pm 0.1), \mathrm{As}_{2} \mathrm{Se}_{m} \mathrm{Te}_{3-m}$ $(m=0,1,1.5,2,3)$, and $\mathrm{As}_{2} \mathrm{~S}_{3}$. ${ }^{11,17}$ The morphology was found to be largely insensitive to substrate choice as long as the substrate is flat and free of contamination. ${ }^{11}$ Exact composition had a much greater influence on domain morphology, with variations away from stoichiometric formulations acting to reduce the domain/trough network. The microscale morphology thus might be related to the mechanical and chemical stabilities of the network. ${ }^{11}$ At stoichiometric compositions, growth of the film might occur by the intrinsic process of island nucleation and columnar growth, resulting in a porous "micronetwork.",

The observation of orpiment nanocrystallites in HRTEM is not at odds with the overall diffraction amorphous nature of the materials as small, isolated, and strained nanocrystallites embedded in a more disordered matrix will give rise to an areally averaged diffraction pattern composed of diffuse rings. From the survey of nanocrystallites in the as-deposited and annealed films, it is difficult to obtain quantitative estimates of the number and size of the nanocrystallites as the only ones detected are the ones that are in a favorable orientation. Moreover, this may not give an insight into the nanoscale heterogeneity of the films as regions that have correlated structures but are not crystallites will not be detected.

A more quantitative estimate of the relative amounts of order can be obtained from FEM where regions of the correlated structure contribute to an increase in the intensity variance regardless of their orientation. Thus we see from the FEM that the nanoscale structure of the as-deposited $\mathrm{As}_{2} \mathrm{~S}_{3}$ film possesses much greater heterogeneity than the annealed film. This higher degree of MRO could be due to a greater number of regions of correlated structure, larger regions, or increased order within them. This is consistent with the model of film nucleation and growth presented by Phillips ${ }^{18}$ whereby the film grows via the addition of "quasistoichiometric" clusters with distorted bonding to already nucleated islands. The material will therefore consist of the quasistoichiometric structurally correlated regions and granular interfaces. The high degree of MRO in the as-deposited films that we detected with FEM is consistent with this as are the mottled contrast in the bright-field image and graininess in intensity in the diffraction pattern. The proximity of such regions of correlated structure and highly distorted intergranular material between them means that the as-deposited film structure occupies a maximum in the energy landscape. Thermal annealing reduces the overall free energy by dissolving the regions of correlated structure, resulting in a more continuous network. This is demonstrated in the FEM results that show a huge reduction in the MRO and no clear structural signature. It is also reflected in the lack of mottled contrast in the bright-field image and the smooth intensity distribution in the selected area diffraction pattern. Such a shift toward a more continuous network structure might also be attended by a density increase, consistent with an upshift in the positions of the diffraction maxima as observed. Indeed, a density increase of $\sim 2 \%$ has been reported. ${ }^{14}$ This relaxation toward a more thermodynamically stable state with less MRO upon annealing was also detected in the seminal work of Gibson and Treacy on thermally evaporated amorphous Ge. ${ }^{19}$ In the previous work by using such thermally evaporated $\mathrm{As}_{2} \mathrm{~S}_{3}$ films to make waveguides, a thermal annealing step is found to significantly reduce the variable etch rate of the $\mathrm{As}_{2} \mathrm{~S}_{3}$ film and the resultant surface roughness. ${ }^{4}$ Our findings support the hypothesis that it is nanoscale heterogeneity in the as-deposited film that is responsible for the variable etch rates.

The FEM results are corroborated by the Raman measurements on the thicker films. The Raman spectrum of the as-deposited film is highly structured with many modes that resemble modes in the crystalline counterparts. Upon annealing the features recede and the Raman spectrum more closely resembles the spectrum of the bulk glass. This change has been interpreted as a reduction in the degree of nanoscale phase separation as the structure tends toward a more thermodynamically stable state. ${ }^{5}$

The structured appearance of the $V(k)$ plot from the asdeposited film indicates that there are particular correlated structures that give rise to the high intensity variance. We note that the maxima in the $V(k)$ plots appear in the same positions as the maxima in the diffraction patterns. This can be readily understood. The regions of correlated structure diffract strongly into a particular $k$; it is thus in this direction that greater variation in the scattered intensity will be detected. At this point it is difficult to address the structures that give rise to such a $V(k)$ signature. It is not valid to simply translate peaks in the diffraction patterns of amorphous materials into interplanar spacings as the diffraction pattern is the sum of all the spatial frequencies in the material, and very often peaks do not correspond to a single real distance. Similarly, for the intensity variance, the $V(k)$ plot arises from the contributions of all the four-body correlations in the material so a simple calculation of interatomic distances is not possible. To interpret the features in the $V(k)$ plot one must use simulations from atomic models. ${ }^{9,20}$ The $V(k)$ plot from the annealed sample is much diminished in intensity and shows no distinct features. This demonstrates that the longer-range atomic correlations that were detected in the as-deposited film have been destroyed by annealing.

The lack of dependence of the $V(k)$ plot on dark-field tilting direction is interesting considering the strong evidence for birefringence in as-deposited $\mathrm{As}_{2} \mathrm{~S}_{3}$ films. This birefringence was found to decay due to room temperature storage (30 days or more), thermal annealing, and irradiation by unpolarized light. ${ }^{21}$ It is thought to arise due to the alignment of small structural units with one direction in the film. ${ }^{21}$ FEM with its sensitivity to higher-order atomic correlations and implemented using one-dimensional dark-field tilting should be sensitive to such structural anisotropy. However, the magnitude of the effect $(0.4 \%)$ could be too small to be detected in this way and the specimens could be too old (at least a month before examination in the TEM) and already have received too great a dose of unpolarized light.

It is interesting that we detect greater MRO in the asdeposited sample using FEM, while it is the annealed sample that displays the FSDP, often considered to be the hallmark of pronounced MRO. Here we must take some care to distinguish between the meaning of MRO measured by FEM and that generally associated with the FSDP. FEM probes 
structural correlations out to a length defined by the instrument resolution. Here we use a small objective aperture and a deliberately low resolution to sample scattered electrons from a volume with lateral dimensions in the range of MRO. The Fourier transform of the objective aperture yields an Airy disk in the object plane, and hence we are probing atomic correlations in the specimen out to approximately the full width at half maximum of the first maximum in the Airy function. In our experimental setup this gives a length of $\sim 1.4 \mathrm{~nm}$. In contrast, the FSDP is generally discussed in terms of a particular structural motif that will reproduce a peak at this position in the diffraction pattern. Generally, this involves motifs that come from the crystalline forms of arsenic and sulfur compounds, ${ }^{12,13}$ such as orpiment $\left(\mathrm{As}_{2} \mathrm{~S}_{3}\right)$ and realgar (AsS) with a range of sizes and correlation lengths.

The origin of the FSDP in terms of a single structural motif is quite controversial and still not well resolved. There are five main candidate structures that have been employed. The first stems from the microcrystallite tradition of modeling amorphous structure and interprets the small width of the FSDP as a measure of crystallite size. The structure of the stoichiometric crystal, orpiment, is used as the interlayer spacing of the orpiment structure roughly coincides with that of the FSDP. However, this approach results in a reduced intensity function that is too highly featured in the high wave vector regions (specifically with the cross-ring correlations from orpiment) and does not reproduce the FSDP very well anyway. $^{22}$ The microcrystallite model is also inconsistent with the fact that the FSDP persists even after the melting temperature of the material has been surpassed. ${ }^{18}$ Another suggested structure along similar lines is that of layers in which the interlayer atomic correlations are stronger than the intralayer correlations. Such a structure mediates the higher wave vector features from the microcrystalline model, yet still has a plausible explanation for the FSDP and fits well with the thermal behavior of this feature and viscosity of the material. ${ }^{23}$ Two cluster models exist for the dominant structural motif in $\mathrm{As}_{2} \mathrm{~S}_{3}$ glasses. The first is the "out-rigger raft" model, which uses a layer structure based on that of orpiment, but slightly reconfigured to obtain a stable molecular cluster. $^{18,24}$ This motif and its four equivalent conformer rafts can well reproduce the features in the Raman spectrum, the FSDP, and its observed dependence on temperature and composition. ${ }^{24}$ The second cluster is the round $\mathrm{As}_{4} \mathrm{~S}_{4}$ molecule, which is the building block of realgar and has been detected in as-deposited films using Raman spectroscopy ${ }^{5}$ and extended $\mathrm{x}$-ray absorption fine structure. ${ }^{25}$ This molecule is stable in the vapor and could plausibly be preserved in the as-deposited film.

Finally, it is important to note that the FSDP can be accounted for without resorting to any crystallite, layer, or cluster. Continuous random network models built using bond-breaking rules and then relaxed to fit the experimental data demonstrate that in both real and reciprocal spaces, the measured curves can be well accounted for by a model that does not contain any layers, rafts, or clusters. ${ }^{26}$ That modeling work strongly suggests that the FSDP does not arise from specific correlations from one dominant motif, but rather from a broad region of real space, perhaps containing a density deficit rather than atomic configurations. ${ }^{26}$ In most recent works, the position of the FSDP has been successfully linked for a range of covalent network glasses to void correlations. In this work, the peak is due to a prepeak in the concentration-concentration Bhatia-Thornton partial correlation function, arising due to chemical short-range ordering of voids around a cation-centered cluster. ${ }^{27,28}$ This is a comparatively short range chemical ordering. The anomalous behavior of this FSDP can be well accounted for by this model. For example, the FSDP increase with temperature is due to increasing numbers of interstitial voids as the material expands. The decrease with pressure can be similarly understood in terms of a decrease in void numbers with increasing density. This also accounts for the diminution of the FSDP after an anneal below the glass transition temperature, which effects material densification. ${ }^{14}$

Our findings can be interpreted using the same model of the FSDP. The as-deposited films contain large regions of correlated structure, that in turn give rise to a lower level of short-range order due to the more disordered, strained material that must exist in the boundaries between the regions. Annealing destroys these energetically unfavorable ordered regions and also provides energy for chemical short-range ordering to occur. Thus overall the MRO measured by FEM decreases, while at the same time the FSDP increases. It is intriguing that previous studies found that the opposite behavior of the FSDP is obtained upon low temperature annealing. We note that the films we are examining are $40 \mathrm{~nm}$ thick, such that every part of the film is only 10-20 times the length scale of MRO away from a surface. Previous studies $^{14,15}$ examining the behavior of thermally evaporated $\mathrm{As}_{2} \mathrm{~S}_{3}$ films with annealing employed films of $1-22 \mu \mathrm{m}$ in thickness deposited onto various substrates. Our study suggests that exact processing conditions, such as film thickness, and perhaps, when the film thickness is low, substrate choice could play a large role. We plan to pursue this question further on thicker specimens and exactly correlate TEM analyses with Raman spectroscopy. At a fundamental level our results suggest that short- and medium-range atomic orderings have a complex interrelationship in the sense that an increase in one does not necessarily imply an increase in the other.

\section{CONCLUSION}

We have presented morphological and structural studies of as-deposited and annealed thermally evaporated $\mathrm{As}_{2} \mathrm{~S}_{3}$ films. Our studies suggest that the $\mathrm{As}_{2} \mathrm{~S}_{3}$ film grows by nucleation and cluster addition, giving rise to the large scale morphological features and nanoscale heterogeneity detected by FEM in the as-deposited film. Annealing the film reduces the MRO and results in a more homogeneous network. The appearance of the FSDP upon annealing seems to suggest a concomitant short-range chemical ordering of voids. Further FEM studies will be coupled to atomic modeling and undertaken on thicker films permitting correlation with Raman spectroscopy to further elucidate the nature of the MRO. 


\section{ACKNOWLEDGMENTS}

The electron microscopy was accomplished at the Electron Microscopy Center for Materials Research at Argonne National Laboratory, a U.S. Department of Energy Office of Science Laboratory operated under Contract No. DE-AC0206CH11357 by UChicago Argonne, LLC. The support of the Australian Research Council through its Federation Fellow and Centre of Excellence programs is gratefully acknowledged.

${ }^{1}$ A. Zakery and S. R. Elliott, J. Non-Cryst. Solids 330, 1 (2003).

${ }^{2}$ A. B. Seddon, J. Non-Cryst. Solids 184, 44 (1995).

${ }^{3}$ R. G. deCorby, N. Ponnampalam, M. H. Pai, H. T. Nguyen, P. K. Dwivedi, T. J. Clement, C. J. Haugen, J. N. McMullin, and S. O. Kasap, IEEE J. Sel. Top. Quantum Electron. 11, 539 (2005).

${ }^{4}$ S. Madden, D.-Y. Choi, D. Bulla, A. Rode, B. Luther-Davies, V. G. Ta'eed, M. D. Pelusi, and D. J. Eggleton, Opt. Express 15, 14414 (2007).

${ }^{5}$ D. G. Georgiev, P. Boolchand, and K. A. Jackson, Philos. Mag. 83, 2941 (2003).

${ }^{6}$ D.-Y. Choi, S. Madden, R. P. Wang, A. Rode, M. Krowlikowska, and B. Luther-Davies, J. Non-Cryst. Solids 353, 953 (2007).

${ }^{7}$ A. C. Y. Liu, R. Arenal, D. J. Miller, X. Chen, J. A. Johnson, O. L. Eryilmaz, A. Erdemir, and J. B. Woodford, Phys. Rev. B 75, 205402 (2007).

${ }^{8}$ X. Chen, J. P. Sullivan, T. A. Friedmann, and J. M. Gibson, Appl. Phys. Lett. 84, 2823 (2004).
${ }^{9}$ M. M. J. Treacy, J. M. Gibson, L. Fan, D. J. Paterson, and I. McNulty, Rep. Prog. Phys. 68, 2899 (2005).

${ }^{10}$ J. M. Cowley, Ultramicroscopy 90, 197 (2002).

${ }^{11}$ C. H. Chen, J. C. Phillips, P. M. Bridenbaugh, and D. A. Aboav, J. NonCryst. Solids 65, 1 (1984).

${ }^{12}$ D. J. E. Mullen and W. Nowacki, Z. Kristallogr. 136, 48 (1972).

${ }^{13}$ A. Kyono, M. Kimata, and T. Hatta, Am. Mineral. 90, 1563 (2005).

${ }^{14}$ E. Marquez, J. M. Gonzalez-Leal, R. Jimenez-Garay, and M. Vlcek, Thin Solid Films 396, 183 (2001).

${ }^{15}$ J. P. de Neufville, S. C. Moss, and S. R. Ovshinsky, J. Non-Cryst. Solids 13, 191 (1973/74).

${ }^{16}$ D.-Y. Choi, S. Madden, A. Rode, R. Wang, and B. Luther-Davies, J. Appl. Phys. 102, 083532 (2007)

${ }^{17}$ C. H. Chen, J. C. Phillips, K. L. Tai, and P. M. Bridenbaugh, Solid State Commun. 38, 657 (1981).

${ }^{18}$ J. C. Phillips, J. Non-Cryst. Solids 43, 37 (1981).

${ }^{19}$ J. M. Gibson and M. M. J. Treacy, Phys. Rev. Lett. 78, 1074 (1997).

${ }^{20}$ A. C. Y. Liu, R. Arenal, and X. Chen, Phys. Rev. B 76, 121401 (2007).

${ }^{21}$ V. I. Mikla and V. M. Kryshenik, J. Non-Cryst. Solids 330, 33 (2003).

${ }^{22}$ A. J. Leadbetter and A. J. Apling, J. Non-Cryst. Solids 15, 250 (1974).

${ }^{23}$ L. Busse, Phys. Rev. B 29, 3639 (1984).

${ }^{24}$ J. C. Phillips, C. A. Beevers, and S. E. B. Gould, Phys. Rev. B 21, 5724 (1980).

${ }^{25}$ R. J. Nemanich, G. A. N. Connell, T. M. Hayes, and R. A. Street, Phys. Rev. B 18, 6900 (1978).

${ }^{26}$ T. G. Fowler and S. R. Elliott, J. Non-Cryst. Solids 92, 31 (1987).

${ }^{27}$ S. R. Elliott, J. Phys.: Condens. Matter 4, 7661 (1992).

${ }^{28}$ S. R. Elliott, Phys. Rev. Lett. 67, 711 (1991). 